\title{
O EQUILÍBRIO DE HEMIPARÉTICOS CRÔNICOS PODE MELHORAR APÓS 12 SEMANAS DE CIRCUITO DE TREINAMENTO?
}

Mileide Cristina Stoco de Oliveira ${ }^{1}$, Andressa Sampaio Pereira ${ }^{2}$, Danilo Bravo de Freitas ${ }^{2}$, Mariana Bósio Mathias ${ }^{2}$, Fabiana Araujo Silva ${ }^{1}$, Carla de Oliveira Carletti', Thiago Kendy Kawano ${ }^{3}$, Vanessa Niens Van Den Broek $^{3}$, Bruna de Mello Padovan ${ }^{2}$, Isabella Cristina Leoci ${ }^{2}$, Tânia Cristina Bofi ${ }^{4}$, Lúcia Martins Barbatto ${ }^{4}$, Augusto Cesinando de Carvalho ${ }^{4}$.

Universidade Estadual Paulista - UNESP, ${ }^{1}$ Programa de Pós-Graduação Residência em Fisioterapia, ${ }^{2}$ Curso de Fisioterapia, ${ }^{3}$ Programa de Pós Graduação de Lato Senso em Fisioterapia, ${ }^{4}$ Departamento de Fisioterapia, Faculdade de Ciências e Tecnologia - FCT, Presidente Prudente, SP. e-mail: cristina.mileide@gmail.com

\section{RESUMO}

A reabilitação após um acidente vascular encefálico é melhorar o desempenho funcional de hemiparéticos. A Fisioterapia em Grupo no formato de Circuit Training (FGCT) utiliza exercícios funcionais específicos com foco na repetição e na progressão contínua de exercícios em um número de estações de trabalho organizadas em um formato de circuito, para melhorar a capacidade funcional. O objetivo desse estudo foi analisar o equilíbrio funcional de hemiparéticos submetidos a doze semanas de tratamento na FGCT. Os hemiparéticos foram avaliados através da Escala de Equilíbrio de Berg (EEB) em uma avaliação inicial (AV1) e outra avaliação final (AV2) após doze semanas de FGCT. Os resultados mostraram que houve uma melhora significativa no equilíbrio dos pacientes que realizaram a FGCT. Concluímos que na FGCT os paciente que realizaram a FGCT tiveram melhora no seu equilíbrio.

Palavras-chave: fisioterapia, acidente vascular encefálico, equilíbrio postural, exercícios em circuitos, reabilitação neurológica.

\section{THE HEMIPARESIS CHRONIC BALANCE CAN IMPROVE AFTER 12 WEEKS TRAINING CIRCUIT?}

\section{ABSTRACT}

The rehabilitation after a stroke is to improve the functional performance of hemiparesis. Physiotherapy Group in Circuit Training format (PGCT) uses specific functional exercises focusing on repetition and continuous progression exercises in a number of workstations arranged in a circuit format, to improve functional capacity. The aim of this study was to analyze the functional balance of hemiparesis submitted to twelve weeks of treatment in PGCT. The hemiparesis were evaluated using the Berg Balance Scale (BBS) on an initial assessment (AV1) and a final evaluation (AV2) after twelve weeks of PGCT. The results showed a significant improvement in the balance of patients who underwent PGCT. We conclude that PGCT the patient who underwent FGCT had improved their balance.

Keywords: physical therapy specialty, stroke, postural balance, circuit-based exercise, neurological rehabilitation.

\section{INTRODUÇÃO}

O acidente vascular encefálico (AVE) é um problema de saúde mundial e uma das principais causas de incapacidade ${ }^{1}$. A sequela mais evidente após o AVE é a hemiparesia sendo caracterizada por deficiência motora, espasticidade e fraqueza muscular no hemicorpo contralateral à lesão, e pode estar associada ao déficit de coordenação motora e equilíbrio, alterações sensitivas, cognitivas, perceptivas e de linguagem ${ }^{2}$, afetando todos os domínios da Classificação Internacional de Incapacidade e Saúde, com limitações de atividades e restrições de participação ${ }^{3}$.

Estudos mostram que apenas $14 \%$ conseguem uma recuperação completa da função física e $25 \%$ a $50 \%$ necessitam de alguma ajuda com as atividades da vida diária ${ }^{4}$. É indiscutível a importância da fisioterapia para estes hemiparéticos que tem uma rotina que varia da internação hospitalar, intervenção cirúrgica até o tratamento fisioterapêutico. A fisioterapia tende a melhorar a desempenho motor a partir da 
reeducação dos movimentos globais e finos, da coordenação, equilíbrio, força muscular, reeducação postural e marcha. Neste sentido são utilizados vários métodos para reabilitação física e funcional destes hemiparéticos ${ }^{5}$.

Após o AVE, quanto mais cedo começar a reabilitação, melhor poderá ser o prognóstico funcional. Na fase aguda a reabilitação é utilizada para conseguir uma melhor recuperação funcional nos primeiros meses e reduzir a deficiência durante os anos subsequentes, utilizando-se principalmente de treinamento de tarefas específicas, visando às necessidades dos hemiparéticos, bem como a prática de alta intensidade com repetições suficientes ${ }^{6}$.

Existem evidências na literatura indicando a recuperação substancial da função motora dos membros inferiores nos primeiros 3 meses pós-AVE seguido por melhorias significativamente menores depois deste período, porém os ensaios experimentais indicam mudanças substanciais na capacidade de andar mesmo em hemiparéticos crônicos quando as intervenções fisioterapêuticas são especificas para a marcha ${ }^{7}$.

Após alta da reabilitação, os hemiparéticos atingem uma deambulação independente, porém apresentam redução de velocidade, distância percorrida, padrões assimétricos e instabilidade postural, contribuindo ainda mais para a condição de sedentarismo. Isso aponta a necessidade da participação desses hemiparéticos crônicos em atividade e treinamento físicos constantemente para melhorar suas condições físicas e funcionais e reduzir o sedentarismo e suas consequências ${ }^{7,8}$.

A inatividade é um dos maiores problemas relacionados com o declínio da mobilidade pós-AVE e programas que evitem o sedentarismo, melhorem a marcha e as atividades funcionais são muito importantes para esses hemiparéticos ${ }^{9}$. O treinamento físico pode promover uma série de efeitos positivos, como a redução da pressão arterial, a diminuição de riscos cardiovasculares, melhora da condição respiratória, redução da mortalidade por doenças coronárias, diminuição das condições de depressão psicológica, sendo ainda, capaz de melhorar a independência e a qualidade de vida, além de amenizar diversos problemas pós-AVE como a fadiga, incidência de quedas e fraturas ${ }^{10}$.

$\mathrm{Na}$ hemiparesia ocorrem alterações nas reações posturais entre o lado parético e o não parético o que contribui para reduzir o equilíbrio e a mobilidade dos indivíduos. A falta de equilíbrio é um fator que desfavorece a realização das atividades de vida diária de um indivíduo pós-AVE, aumentando o risco de quedas e implicando diretamente na qualidade de vida ${ }^{11,12}$ e portanto os exercícios terapêuticos são benéficos em qualquer fase da hemiparesia.

Há evidências que a fisioterapia de grupo em circuito de treinamento (FGCT) é capaz de melhorar a capacidade funcional de hemiplégicos. É um modelo de terapia que utiliza exercícios e atividades ativas funcionais específicas realizadas de forma intensiva em uma configuração de grupo e com foco na repetição e progressão contínua da intensidade dos exercícios numa série de estações de trabalhos dispostos em um circuito dirigido visando melhorar a mobilidade geral, a capacidade de andar, a coordenação, o equilíbrio funcional ${ }^{13-16}$. Este trabalho objetivou analisar o equilíbrio funcional de hemiparéticos crônicos submetidos à FGCT por 12 semanas.

\section{METODOLOGIA}

Para realização deste estudo clínico foram recrutados e inclusos indivíduos com hemiparesia unilateral por AVE, com tempo de lesão $\geq$ há 12 meses, em atendimento fisioterapêutico, capazes de realizar a marcha com ou sem auxílio de órtese, ter habilidade para realizar teste de caminhada e ausência de déficits cognitivos avaliados pelo mini exame do estado mental (MEEM) ${ }^{17}$ (ponto de corte para indivíduos analfabetos $18 / 19$ e para indivíduos com instrução escolar 24/25), alteração do tônus dos músculos extensores do tornozelo do lado parético com a pontuação diferente de zero na escala de Ashworth Modificada ${ }^{18}$. Todos os voluntários foram informados sobre os objetivos e procedimentos do estudo e após concordarem com sua participação assinaram um termo de consentimento livre e esclarecido. $O$ estudo foi aprovado pelo Comitê de Ética da FCT-UNESP (CAAE: 45076015.3.0000.5402)

Foi realizada uma entrevista individual para coleta de dados e a seguir foi realizada avaliação do equilíbrio funcional utilizando a Escala de Equilíbrio de Berg (EEB) que consiste em 14 tarefas dinâmicas e estáticas. Os itens avaliados recebem uma pontuação de 0 a 4 baseadas na habilidade para realizar as tarefas propostas. A pontuação ' 0 ' representa inabilidade para completar um item e a pontuação '4' representa a habilidade de completar a tarefa de 
modo independente. $O$ teste totaliza 56 pontos. Abaixo de 36 pontos o risco de quedas é de $100 \%$, ao passo que, com o aumento dos pontos o risco de queda é cada vez menor ${ }^{17}$.

A primeira avaliação foi denominada de AV1 e a segunda de AV2 que ocorreu após os hemiparéticos serem submetidos a 12 semanas de FGCT. O modelo de $\mathrm{FGCT}^{13,19}$ consistiu na prática de exercícios em 10 estações diferentes. As estações foram montadas em espaços com graus de dificuldades diferentes na realização do exercício utilizando bastões, cadeiras, escada e rampa e cones: 1a Estação: Uma pequena plataforma quadrada de madeira medindo $45 \mathrm{x}$ $45 \times 15 \mathrm{~cm}$ colocada no chão onde indivíduo na posição ortostática deve elevar a perna de modo a colocar alternadamente os pés sobre a mesma. 2a Estação: Três círculos de $15 \mathrm{~cm}$ formando um triângulo são colados na parede a $110 \mathrm{~cm}$ do chão. Na posição sentada o paciente deve levar suas mãos entrelaçadas em direção a cada um deste círculo. 3a Estação: Três círculos de $15 \mathrm{~cm}$ formando um triângulo são colados no chão. $\mathrm{Na}$ posição ortostática o paciente deve tocar com o bastão cada círculo, sempre movendo anteriormente o tronco. $O$ paciente é mantido a $50 \mathrm{~cm}$ do triângulo formando pelos círculos. 4a Estação: Quatro cones dispostos em linha reta são colocados no chão. $O$ paciente deve fazer 0 caminho cruzando cada cone e ao chegar ao final, voltar pelo lado externo em linha reta. 5a Estação: Três círculos de $15 \mathrm{~cm}$ formando um triângulo são colados na parede a $220 \mathrm{~cm}$ do chão. $O$ paciente deve tocar cada círculo utilizando um bastão com as mãos entrelaçadas. 6a Estação: Cadeiras sem braços são posicionadas para o paciente sentar e levantar. 7ạ Estação: Um corrimão localizado em frente ao paciente. $\mathrm{Na}$ posição ortostática segurando o corrimão o paciente deve agachar até o chão e retornar para a posição inicial. 8a Estação: Um corrimão localizado em frente ao paciente. $\mathrm{Na}$ posição ortostática segurando o corrimão o paciente deve andar lateralmente ritmicamente. 9ạ Estação: Uma escada de madeira e 5 degraus que termina numa plataforma de $90 \times 90 \mathrm{~cm}$ e seguida inicia uma rampa de 6 graus de inclinação. $O$ paciente deve subir pela escada e descer pela rampa e vice versa. 10 astação: uma cadeira onde o paciente e segura um bastão com as mãos entrelaçadas e faz o movimento de rotação de tronco associado com extensão e flexão de ombro simulando o movimento de remada.

O tempo em que os hemiparéticos ficaram em cada estação foi de 3 minutos e ao final trocavam de estações, ou seja, quem estava na estação número 1 era encaminhado para a estação número 2 e assim sucessivamente. A duração de cada sessão foi de 60 minutos e ocorreram duas vezes na semana durante 12 semanas.

Os dados coletados foram alocados em planilhas e para o tratamento estatístico foi utilizado o teste de Shapiro-Wilk para testar a normalidade do conjunto de dados e, com base nos parâmetros fornecidos, foi realizada a análise descritiva e os dados foram apresentados em valores de média e desvio-padrão. Todas as análises foram realizadas por meio do software estatístico BioEstat (versão 5.0). O nível de significância foi estabelecido em $5 \%$.

\section{RESULTADOS}

A amostra do estudo foi constituída de 19 hemiparéticos. Destes 10 eram do sexo masculino e 9 do sexo feminino, com idade média de $60,05 \pm 12,09$ anos. A hemiparesia direita foi observada em 10 indivíduos e a esquerda em 9 indivíduos.

A análise dos valores da AV1 do EEB demonstrou que os hemiparéticos marcaram $43,32 \pm 10,06$ pontos. Na AV2 marcaram 46,79 \pm 6,12 pontos (Figura 1). A análise estatística demonstrou diferença significativa entre os valores de AV1 e AV2 $(r=-1,73 ; p<0,05)$. 


\section{Valores de EEB na avaliação incial e final}

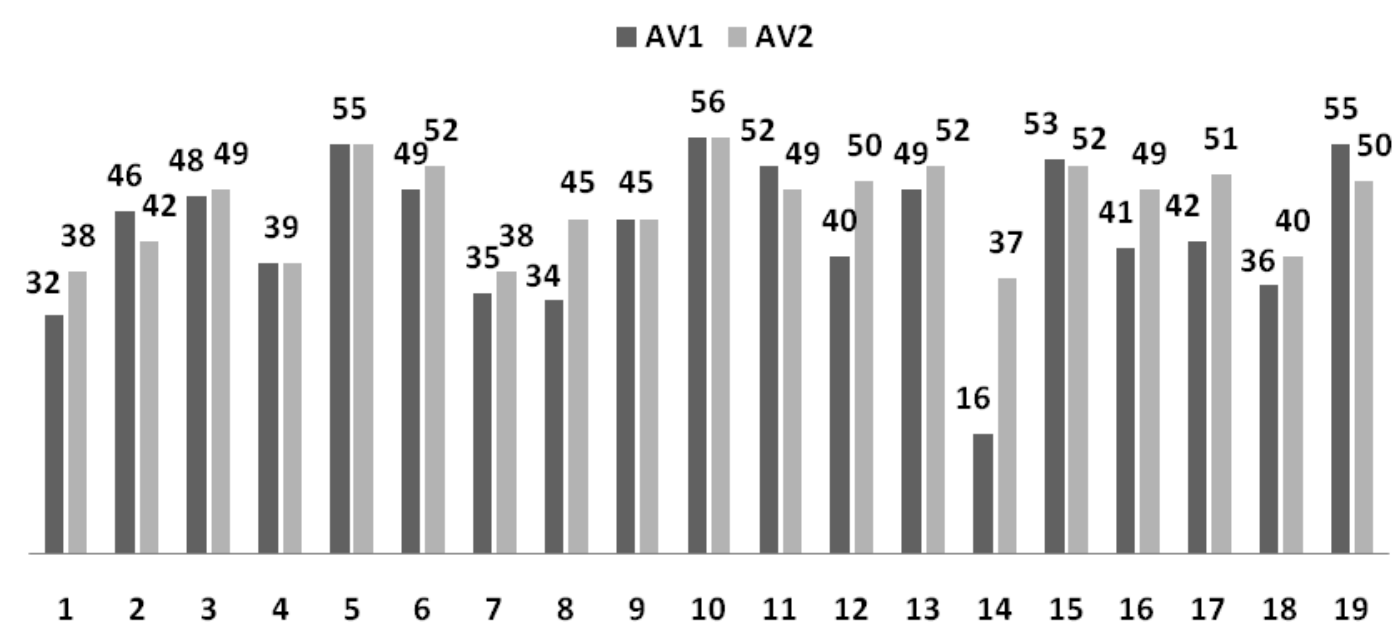

Figura 1. Dados do EEB na avaliação inicial (AV1) e final(AV2) da amostra.

\section{DISCUSSÃO}

Os resultados desse estudo mostraram que o modelo de FGCT utilizado melhorou o equilíbrio de hemiparéticos. Este modelo de terapia em grupo tem sido proposto para aumentar a quantidade de tempo em com tarefas específicas para melhorar a capacidade funcional de hemiparéticos ${ }^{12,20}$. Outros benefícios desta terapia incluem o apoio dos pares e inter-relação social bem como a economia de custos para o sistema de saúde ${ }^{21}$.

Este estudo possibilitou que hemiparéticos vivenciassem um modelo de terapia diferente da terapia convencional, além disso, possibilitou aos autores colocarem em prática as teorias observadas na literatura e ampliar as possibilidades terapêuticas para hemiparéticos crônicos que muitas vezes ficam em suas casas mantendo um comportamento sedentário.

Os resultados deste estudo corroboraram com outros encontrados, demonstrando que os exercícios específicos realizados em forma de FGCT podem promover a melhora no equilíbrio, da resistência de marcha e na capacidade funcional dos pacientes hemiparéticos crônicos, uma vez que foram obtidos um resultado favorável no score da EEB após o período de treinamento ${ }^{22-23 .}$

Como no estudo de Kim et al. $^{22}$, que mostra uma melhora nos valores do EEB pré e pós-intervenção, pois na pré foi de $44,5 \pm 3,8$ e pós-intervenção de $51,1 \pm 2,52$. Assim como, o estudo de Scalzo et al. ${ }^{23}$ que nos seus resultados apontou uma melhora no teste de EEB, dado que os resultados pré-intervenção foram 43,6 $\pm 6,5$ e pós-intervenção $48,5 \pm 5,7$. Para esse estudo é importante ressaltar que a maior parte dos participantes tiveram um aumento igual ou superior a 3 pontos na EEB.

A experiência alcançada com este modelo terapêutico nos permite inferir que os hemiparéticos crônicos têm uma maior possibilidade de melhorar sua mobilidade e autonomia funcional.

\section{CONCLUSÃO}

Os resultados de deste estudo demonstraram a melhora no equilíbrio funcional de hemiparéticos crônicos submetidos à FGCT por 12 semanas.

\section{CONFLITO DE INTERESSES}

Os autores declaram não haver qualquer potencial conflito de interesses que possa interferir na imparcialidade deste trabalho científico.

\section{REFERÊNCIAS}

1. Dobkin BH. Rehabilitation after stroke. N Engl J Med. 2005;352(16):1677-84. DOI: http://dx.doi.org/10.1056/NEJMcp043511

2. Brogardh C, Lexell J. Effects of cardiorespiratory fitness and muscle-resistance training after stroke. PMR. 2012;4(11):901-7. DOI: http://dx.doi.org/10.1016/j.pmrj.2012.09.11 57

3. Buchalla CM. Classificação Internacional de Funcionalidade, Incapacidade e Saúde. São Paulo: Universidade de São Paulo - EDUSP; 2008. 
4. Gordon NF, Gulanick M, Costa F, Fletcher G, Franklin BA, Roth EJ et al. Physical activity and exercise recommendations for stroke survivors: an American Heart Association scientific statement from the Council on Clinical Cardiology, Subcommittee on Exercise, Cardiac Rehabilitation, and Prevention; the Council on Cardiovascular Nursing; the Council on Nutrition, Physical Activity, and Metabolism; and the Stroke Council. Circulation. 2004;109(16):2031-41. DOI: http://dx.doi.org/10.1161/01.CIR.0000126280.65 777.A4

5. Veerbeek JM, van Wegen $E$, van Peppen $R$, van der Wees PJ, Hendriks E, Rietberg M, et al. What Is the Evidence for Physical Therapy Poststroke? A Systematic Review and Meta-Analysis. Plos One. 2014;9(2):e87987. DOI: http://dx.doi.org/10.1371/journal.pone.0087987 6. Van de Port IGL, Wevers LEG, Lindeman E, Kwakkel G. Effects of circuit training as alternative to usual physiotherapy after stroke: randomised controlled trial. Brit Med J. 2012;344:e2672.

DOI:

http://dx.doi.org/10.1136/bmj.e2672

7. Hornby TG, Straube DS, Kinnaird CR, Holleran $\mathrm{CL}$, Echauz AJ, Rodriguez KS et al. Importance of specificity, amount, and intensity of locomotor training to improve ambulatory function in patients poststroke. Top Stroke Rehabil. 2011;18(4):293-307.

DOI:

http://dx.doi.org/10.1310/tsr1804-293

8. Donovan K, Lord SE, McNaughton HK, Weatherall M. Mobility beyond the clinic: the effect of environment on gait and its measurement in community-ambulant stroke survivors. Clin Rehabil. 2008;22(6):556-63. DOI: http://dx.doi.org/10.1177/0269215507085378

9. Wevers L, van de Port I, Vermue M, Mead G, Kwakkel G. Effects of Task-Oriented Circuit Class Training on Walking Competency After Stroke A Systematic Review. Stroke. 2009;40(7):2450-9. DOI:

http://dx.doi.org/10.1161/STROKEAHA.108.5419 46

10. Brogardh C, Lexell J. Effects of cardiorespiratory fitness and muscle-resistance training after stroke. PM\&R. 2012;4(11):901-7. DOI: http://dx.doi.org/10.1016/j.pmrj.2012.09.11 57

11. Pompeu SMAA, Pompeu JE, Rosa M, Silva MR. Correlação entre função motora, equilíbrio e força respiratória pós Acidente Vascular Cerebral. Rev Neurocienc. 2011;19(4):614-20.
12. Park KT, Kim HJ. Effect of the a circuit training program using obstacles on the walking and balance abilities of stroke patients. J Phys Ther Sci. 2016;28(4):1194-8.

DOI: http://dx.doi.org/10.1589/jpts.28.1194

14. English CK, Hillier SL, Stiller KR, Warden-Flood A. Circuit class therapy versus individual physiotherapy sessions during inpatient stroke rehabilitation: A controlled trial. Arch Phys Med Rehab. 2007;88(10):1364-8. DOI: http://dx.doi.org/10.1016/j.apmr.2007.04.010 15. English C, Hillier S. Circuit class therapy for improving mobility after stroke: a systematic review. J Rehabil Med. 2011;43(7):565-71. DOI: http://dx.doi.org/10.2340/16501977-0824

16. Kaur G, English C, Hillier S. How physically active are people with stroke in physiotherapy sessions aimed at improving motor function? A systematic review. Stroke Res Treatment. 2012;19. DOI: http://dx.doi.org/10.1155/2012/820673

17. Miyamoto ST, Junior L, Berg KO, Ramos LR, Natour J. Brazilian version of the Berg balance scale. Braz J Med Biol Res. 2004;37(9):1411-21. DOI: $\quad$ http://dx.doi.org/10.1590/S0100879X2004000900017

18. Bertolucci PHF, Brucki SMD, Campacci SR, Juliano Y. The Mini-Mental-State-Examination in an outpatient population - influence of literacy. Arq Neuro-Psiquiat. 1994;52(1):1-7. DOI: http://dx.doi.org/10.1590/S0004-

282X1994000100001

19. Gregson JM, Leathley M, Moore AP, Sharma AK, Smith TL, Watkins CL. Reliability of the Tone Assessment Scale and the modified Ashworth scale as clinical tools for assessing poststroke spasticity. Arch Phys Med Rehabil. 1999;80(9):1013-6. DOI: http://dx.doi.org/10.1016/S0003-9993(99)900539.

20. Ada L, Mackey F, Heard R, Adams R. Stroke rehabilitation: does the therapy area provide a physical challenge? Aust J Physiother. 1999;45:33-38.

21. Dean CM, Richards CL, Malouin F. Taskrelated circuit training improves performance of locomotor tasks in chronic stroke: a randomized, controlled pilot trial. Arch Phys Med Rehabil. 2000;81(4):409-417.

DOI: http://dx.doi.org/10.1053/mr.2000.3839

22. Kim B, Park Y, Seo Y, Park S, Cho H, Moon $H$, Lee $H$, Kim M, Yu J. Effects of individualized versus group task-oriented circuit training on balance ability and gait endurance in chronic stroke inpatients. J Phys Ther Sci. 2016; 
28(6):1872-5.

DOI:

http://dx.doi.org/10.1589/jpts.28.1872

23. Scalzo PL, Zambaldi PA, Rosa DA, Souza DS, Ramos TX, Magalhães V. Effects of a specific balance training in chronic hemiplegic. Efeito de um treinamento específico de equilíbrio em hemiplégicos crônicos. Rev Neurocienc 2011;19(1):90-7.

Recebido para publicação em 19/08/2016

Revisado em 05/09/2016

Aceito em 12/09/2016 